\title{
Business Process Modelling as a Critical Success Factor in Implementing an ERP System
}

Mojca Indihar Štemberger, Vesna Bosilj Vukšić, Andrej Kovačič̌

Abstract:

Many enterprises are adopting enterprise resource planning (ERP) systems for improving their efficiency and productivity. Although there are tremendous benefits to implementing an ERP system, there are just as many risks. The problem is that many projects of this type are unsuccessful, mostly due to their complexity being underestimated. The purpose of the paper is twofold: (1) to analyse the critical success factors (CFSs) of ERP implementation projects and (2) to propose a framework based on a composite approach to ERP systems implementation. A special emphasis is given to business process modelling, because the key to the successful choice, implementation and usage of an ERP system is the fit of planned processes in an organisation with processes implemented in the solution. The paper also presents some results of empirical investigation in the field of ERP implementation.

Keywords: enterprise resource planning (ERP) systems, critical success factors (CFSs), implementation, framework, business process modelling

JEL: M15 - IT Management

DOI: $10.2478 / v 10033-009-0014-3$

\section{Introduction}

An Enterprise Resource Planning (ERP) system is a business management system comprising integrated sets of comprehensive software that can be used, when successfully implemented, to manage and integrate all business processes and functions within an organisation (Shehab et al., 2004, Ehie and Madsen, 2005). They usually include a set of mature business applications and tools for financial and cost accounting, sales and distribution, management of materials, human resources, production planning and computer integrated manufacturing, supply chain, and customer information (Boykin, 2001; Chen, 2001, Koh and Saad, 2006, Motwani et al., 2005).

Potential benefits of implementing ERP systems include drastic declines in inventory, breakthrough reductions in working capital, abundant information about customers' wishes and needs, along with the ability to view and manage the extended enterprise of suppliers, alliances and customers as an integrated whole (Chen, 2001; Shehab et al., 2004). Among the most important characteristics of ERP systems that can bring these benefits (Nah et al., 2001; Soh et al., 2000) are their abilities to: automate and integrate business processes across organisational functions and locations; enable the implementation of all variations of best business practices; share common data and practices across the entire enterprise.

Since ERP is believed to provide an organization with several benefits, organisations increasingly implement their information systems (IS) by purchasing ERP systems. Over $80 \%$ of the Fortune 500 companies had adopted ERP systems by 2004 (META Group, 2004). Recently, numerous
*Mojca Indihar Štemberger
University of Ljubljana, Faculty of Economics
e-mail: mojca.stemberger@ef.uni-lj.si

\section{Vesna Bosilj Vukšić}
University of Zagreb, Faculty of Economics and
Business - Zagreb
e-mail: vbosilj@efzg.hr

\section{Andrej Kovačič}
University of Ljubljana, Faculty of Economics e-mail: andrej.kovacic@ef.uni-lj.si 
small and medium sized enterprises (SMEs) have started to adopt ERP systems (Loh and Koh, 2004). The potential benefit of successfully implementing an ERP system is large, and even critical to organisational performance and survival (Woo, 2007). However, while some companies have achieved great benefits from their ERP systems, others have experienced failures. They underestimated the complexity of such projects in many cases, and as a consequence the rate of unsuccessful projects was high (Al-Mashari, 2003). Therefore, one of the most common reasons for failure, is still a fact that was recognised almost a decade ago, namely that the logic of the system may conflict with the business processes in an organisation (Davenport, 1998). The situation is similar to that in the implementation of CRM, SCM or e-business concepts.

Traditional ERP implementation methodologies provided practitioners with a list of the activities that have to be conducted in a software implementation project. These methodologies evolved into a set of "recommended collection of phases, procedures rules, techniques, tools, documentation, management and training used to develop a system (Avison and Fitzgerlad, 2003). However, the typical enterprise implementation environment is highly complex and risky.

The purpose of the paper is to expose and analyse the critical success factors of ERP implementation projects and to propose a framework that would enable the management of their complexity and lead to their successful outcome. The framework is based on a composite approach to ERP systems implementation. A special emphasis is given to business process modelling, because we believe that the key to a successful choice, implementation and usage of an ERP system is the fit of planned processes in an organisation with processes implemented in the solution.

The structure of the paper is as follows: Section 2 presents a review of critical success factors by ERP implementation projects. Section 3 describes several possibilities and some results of an empirical investigation are presented. Section 4 describes the proposed framework that can lead to a successful ERP implementation. The role of business process modelling is each phase is analysed. The last section concludes the paper and gives several directions for further research.

\section{Critical success factors of ERP systems implementation}

Recently, numerous authors investigated critical success factors (CFSs) for ERP implementation. In the literature, ERP teamwork and composition, change management program and culture, top management support, project management and BPR with minimum customization are the four most often cited critical factors (Nah et.al, 2003, A-Mashari, 2003, Garcia-Sanchez and Perez-Bernal, 2007).

The reason for the many failures that have occurred is that companies have concentrated exclusively on the technical aspects while ignoring the changed management elements. The main reason of failure is underestimation of the complexity of such project that requires several organisational changes and involvement of employees (Huang et al, 2003). Mc Adam and Galloway (2005) explore the organisational issues involved in implementing an ERP. Their findings indicate that ERP should be incorporated within a wider organisational change programme. Huang et. al (2004) used a Delphi method to identify potential ERP project risk factors. Among the synthesized 28 risk factors, the authors summarized the top ten risk factors that affect the ERP projects more than others. The risk to fail to redesign business processes was ranked ninth.

The lack of appropriate cultural and organisational readiness was found as the most important factor contributing to the failure of an ERP project (Gargeya, Brady, 2005). According to Woo (2007), when an attempt is made to adapt the implementation of ERP to Chinese culture, management and company style, the implementation is successful. The research conducted by Garcia-Sanchez and Perez-Bernal (2007) implies that cultural aspects are a likely cause of the differences in the ordering of CSF priority levels in different world regions. Using a case study methodology, Motwani et.al (2005) suggests that careful change management, network relationships and cultural readiness have a positive impact on ERP implementations.

Massive organisational changes involved in ERP implementation result from the shift in a business design from a fragmented, functional-based organisational structure to a process-based one (Davenport, 1998). The alignment of the standard ERP processes with the company's business processes has for a long time been considered a critical step of the implementation process. Some organisations do not even know which processes 
they have. Others think that the best practice implemented in these solutions will be applied to their processes automatically. A project could therefore be lengthy, inconsistent, exceed the budget, or may result in incomplete installations of system modules and consequently lower benefits than hoped for (Al-Mashari, 2003). There are usually three major parties involved in an ERP system implementation: the organization implementing the system (the implementer), the organization that developed the ERP system (vendor) and an organization aiding the implementation (the consultant). Each of these parties contributes in different ways to the project. According to Hains and Goodhue (2003), the implementer has the detailed knowledge of its own particular business processes and organizational context, which is essential for successful implementation. Bozarth (2006) points out the importance of involving key users in the specification and selection process, especially when the new system promises radical change to the current processes.

\section{How to implement an ERP system successfully?}

The implementation of ERP systems has been one of the first problems addressed by the literature on the subject because not all companies have been successful in their implementations. One of the disadvantages is relatively high costs, particularly in the case of a more significant gap between the processes in an organisation and the processes implemented in the ERP system. Another disadvantage is the threat of losing competitive advantage, which can lie in flexibly customised business processes better suited to an organisation as its "best practice". Potential impediments derive from functional coordination problems related to inadequate support from functional units and coordination among functional units, project management related to business process change, and change management related to resistance of users (Kim et al., 2005). In this section we present a proposal for the framework that can help organisations to avoid the difficulties mentioned above and to implement an ERP system successfully.

\subsection{Alternative possibilities for the selection and implementation of an ERP system}

It is very important to select an appropriate ERP system. Since there are several ERP systems on the market, it is difficult to select the right one. The decision concerning the purchase of individual modules or their development can be done only on the basis of good knowledge. Process mining is introduced as a preliminary step in ERP implementation (van der Aalst and Weijters, 2004). It is very important to achieve a good alignment between ERP modules and supported business processes. Very often, ERP solutions should be customized and aligned to customers' requirements. On the other hand, companies that implement ERP systems have the opportunity to redesign their business practices using templates imbedded in the software. Although sometimes seen as large IT projects, ERP projects are in fact business process change projects in which core organizational business processes are changed to align with the best practices, business process rules and procedures defined during ERP implementation activities. Companies seldom implement all of their business processes using a single ERP product. In fact, companies have business processes enabled by many systems, sometimes even by multiple-enterprise systems (Frye and Gulledge, 2007). Figure 1 shows alternative possibilities.

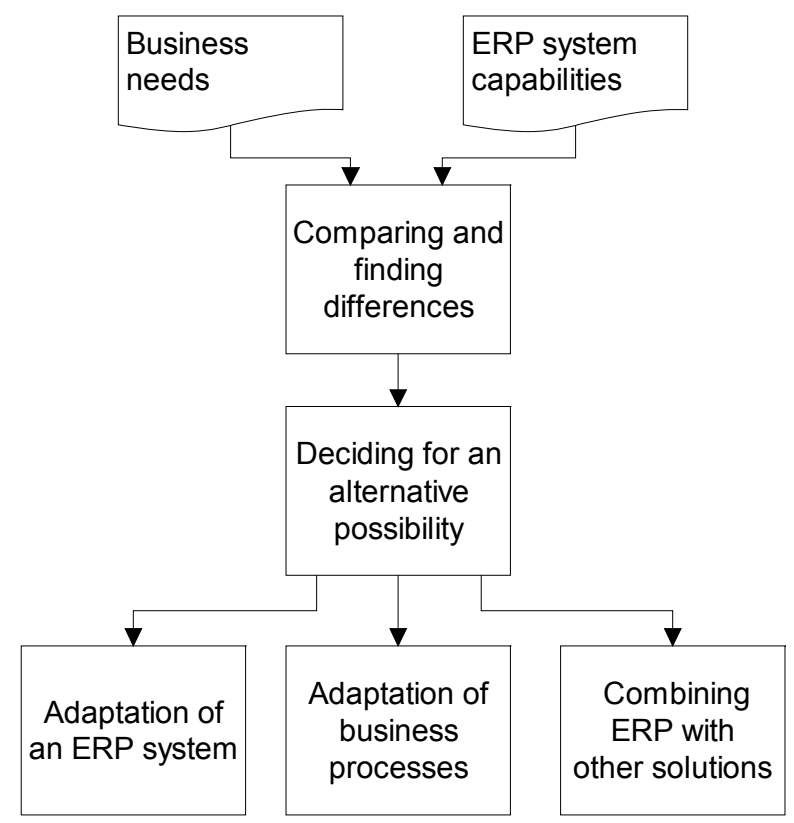

Figure 1: The alternatives for selection and implementation of ERP system

A business first needs to be compared with the capabilities of an ERP system in order to find differences (Kovačič, Bosilj-Vukšić, 2005). Business needs are best described by the organisation's strategy and desired business processes. Processes inside an organisation have 
to be compared with reference to the models of an ERP system.

The problem is that many organisations fail to make such a comparison at all. In an empirical research study conducted among $\mathrm{ClO}$ s of the 150 largest Slovenian companies at the beginning of 2006, we found that almost half had not assessed their information needs by any kind of business modelling (processes, organisation, data).

After this step an organisation has three alternatives: (1) to adapt an ERP system to their business processes; (2) to adapt their business processes to the processes implemented in an ERP system; (3) to combine the acquired (ERP), integrated (best-of-breed) and engineered (adapted or built) applications. In practice there is also a fourth alternative, namely to do no or no significant adjustment. This alternative surely leads to "living with problems", which means that an organisation uses an ERP system that is not tailored to the way its business is done.

The first alternative is appropriate for those organisations that believe their business processes are better than those implemented in an ERP system and do not want to lose their competitive advantage. Although fine tuning of an ERP system can be done through parameterisation, an additional computer code development is necessary for greater changes. This alternative can cause high additional costs, because customisation of ERP modules can be very expensive. In addition, it presents difficulties in maintenance and upgrading to new versions. As a consequence this alternative can lead to the failure of a project. It would probably be better for such organisations to develop their own solutions in the first place. Nevertheless, ERP vendors do not hesitate adapting their software because they expect high earnings and because such project is easier to manage from their point of view.

The second alternative, also named a technology driven approach (Arif et al., 2005), is the adaptation of business processes to an ERP system. It means that best practices implemented in these software packages have to be applied in an organisation. Although it is theoretically the best way that allows an organisation to reap all possible advantages from an ERP system, such changes are very hard to implement in practice. It means that an ERP implementation project has to include a process redesign project. It makes the situation much more complicated. Many vendors are concerned about the complexity and therefore the high threat of failure. In addition, an organisation might lose advantage of unique and perhaps better business practice.

The best possible alternative in the majority of cases proved to be a composite approach, i.e. a blend of acquired (ERP), integrated (best-of-breed) and engineered (adapted or built) applications. It seems best for standard business processes (e.g. accounting processes) to adapt to best practices, whereas customised business processes (e.g. order fulfilment) should in many cases adapt to an ERP system. It is also possible to combine an ERP solution with best-of-breed or custom developed modules (Stolovitsky, 2006). Furthermore, this alternative is in accordance with the current trends in the ERP market. While vendors propagated usage of monolithic solutions in the past, currently they have switched their tendency to combining their software with industry-specific solutions (Genovese, 2005).

The abovementioned research study also showed that the situation in this area is a source of concern. Only $10 \%$ of companies that had already implemented an ERP system adapted their business processes to the solution, while $9 \%$ adapted it to the software. Another $40 \%$ of organisations decided for the third alternative, which finding is encouraging. However, $41 \%$ of companies have not done anything, which ranks them among those that "live with problems" and make their projects unsuccessful.

Another interesting result of the research study arose from documenting the changes in an ERP system and/or business processes. In $41 \%$ of companies, these changes are not documented at all and in 39\% they are documented only partly. Again, business process modelling would be a very appropriate way to document changes.

\subsection{The framework for the third alternative}

As discussed above, we advise most organisations to apply the third alternative, the composite approach to ERP implementation. In this case some business processes are adapted to the ERP system and in others custom developed or best-of-bread modules are applied. For this alternative we propose the framework presented in Figure 2. It has seven stages that are dependent from each other and are not necessarily carried out in sequence.

\section{(1) Assessing the current situation in an organisation.} In this stage current business processes in an 
organisation are modelled. Several organisations already have some models; however, in practice they rarely correspond to the actual business processes or include an adequate level of details. Therefore, some modelling has to take place in almost every case. Nevertheless there is no need to develop detailed models because such a project could be very lengthy

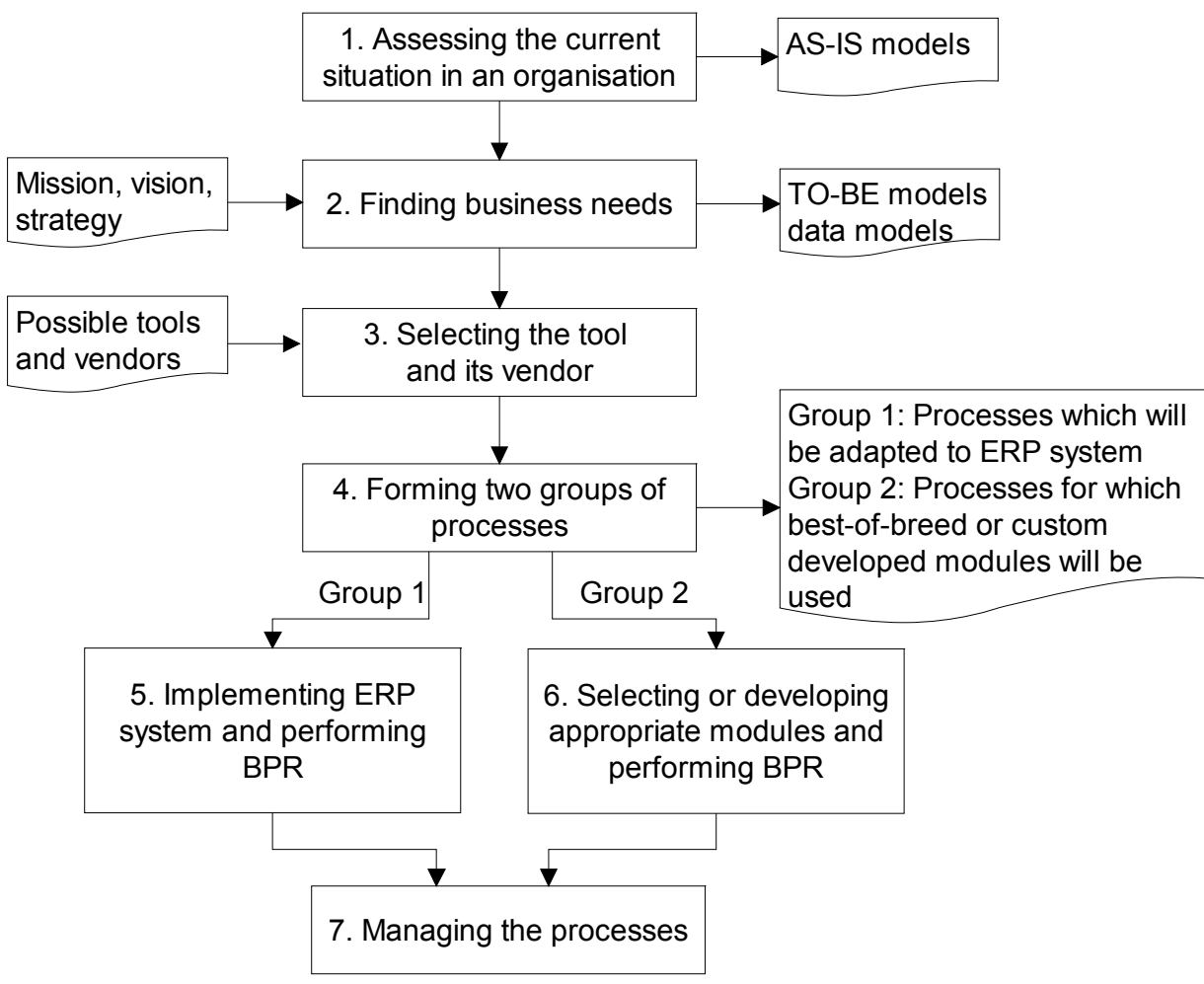

Figure 2: The proposed framework for a composite approach to ERP implementation
2005). One of the most important criteria is matching between business needs and system capabilities. A comparison is possible because business needs are expressed in the form of TO-BE models, which are compared with the reference models of an ERP system. Stages 2 and 3 can also be executed simultaneously. If an organisation has already decided on a particular ERP solution, then it is good if its vendor takes part in phase 2 , because best practices of the solution can be applied to designing TO-BE models.

(4) Forming two groups of processes. The next stage is extremely important. Processes are divided into two groups: the first group consists of the processes that will be adapted to an ERP system, while the second processes are those for which best-of-breed or custom developed modules will be applied. Standard support processes, like processes in accounting, typically fall into the first group. The situation is much more complicated for core processes. On the one hand, and may incur much cost. The results of this stage are high-level AS-IS models of business processes.

(2) Finding business needs. In the second stage business needs are determined in the form of highlevel TO-BE models and data models. The mission, vision and strategy of an organisation are used as an input to this stage. There are various levels of changes from AS-IS to TO-BE processes. Some companies need only small changes, but for others major changes in business processes are necessary. In the latter case the probability of project failure is much higher and as a consequence a higher level of top management involvement is required.

(3) Selecting the tool and its vendor. In stage 3 possible ERP systems on the market and their vendors have to be analysed. The tool has to be selected on the basis of several criteria, some of which are discussed in the literature, e.g. (Wei et al., an organisation might be building competitive advantage of their uniqueness and flexibility. In this case it is better to place them in the second group. On the other hand, a company can acquire a better business process model from an ERP system. Regardless, in making the decision a company has to consider the higher costs of these modules and the fact that vendors are less experienced in their implementation, especially in the servicing sector.

(5) Implementing an ERP system and performing BPR. For the processes in the first group, the selected ERP system is implemented at this stage. The implementation interweaves with a business process change project. In many cases the changes are remarkable. In such cases all the constituent parts of a BPR project, like change management, changes in organisational structures and organisational culture, have to take place. Such project might be extremely 
complex and difficult to manage. It requires a high level of top management and all other employees' involvement. However, if only standard modules of an ERP system are implemented, then the project is usually not so complex.

(6) Selecting or developing appropriate modules and performing BPR. For the processes in the second group, best-of-bread or custom developed modules are implemented. If an appropriate solution to a particular process is available, it has to be implemented and integrated with the rest of the system. In case of custom developed modules, additional modelling of business processes and data is required, because in the initial stages of methodology only high-level models are developed. This stage also requires a business process change with the same characteristics as described in the previous paragraph. Stages 5 and 6 are typically executed at the same time and are tightly connected.

A business process change is performed simultaneously for both phases.

(7) Managing the processes. The last stage actually never ends. It includes the measurement and continuous improvement of business processes. The changes in processes have to be constantly documented. It is important that processes are flexible enough to enable a company to accommodate them to market expectations fast enough.

\subsection{Suggestions for the first and second alternatives}

During ERP systems implementation business process modelling is necessary also for the first (adaptation of an ERP system) or the second (adaptation of business processes) alternative described in the previous section.

Stage 1, in which high-level AS-IS models are developed is inevitable, because an ERP system cannot be selected if it is not known precisely how business processes are performed. Nevertheless, the required level of precision is not high, and high level models are an even better form detailed for this purpose.

Stage 2 can rarely be avoided as well, because in practice no company performs their business processes perfectly. In our experience, the unification of processes (e.g. order fulfilment in different business units) is needed. Without this the software cannot be implemented. For the second alternative reference models of an ERP system play the role of TO-BE models.
In case an organisation decides to adapt their business processes to an ERP system, it has to be selected (stage 3) even more carefully. In stage 4 all processes fall into the first group. Stage 5 is performed as described above and stage 6 is not performed at all or only in a limited scope for the processes that are not covered by the selected solution. In stage 7 making changes to the processes is usually more complicated.

For the first alternative (adaptation of an ERP system), which is not recommendable, yet is, however, quite popular in practice, the situation is somewhat different. In this case the changes of an ERP solution have to be well documented. Again business process modelling can serve as a suitable technique. Business process change takes place for this alternative only in a limited amount, if at all. It reduces the complexity of the project, but as a consequence stage 7 is much more problematic. Of course stage 3 is extremely important as well.

\section{Discussion and conclusion}

ERP projects are more about changing a company's business processes than information technology. Therefore, any company that is not prepared to change its business processes will achieve no real improvement in performance and competitiveness. The research literature on ERP systems confirms that many ERP implementations have been unsuccessful because companies vastly undervalued the importance of reengineering business processes.

This paper analysed some challenges of ERP implementation projects and described their possible alternatives. The authors share the opinion that the best alternative for a majority of companies is the third alternative, which combines adaptation of some business processes to an ERP system with the application of custom developed or best-of-bread modules. As can be concluded from the results of the empirical investigation presented in section 3, several companies have already recognised the benefits of this approach.

This paper presented the framework for a composite approach to ERP implementation that enables successful implementation. Business process modelling plays an important role, especially in the first two stages of the framework because it gives the answers to a set of questions, such as:

- How to ensure that ERP system is in compliance with company's end-to-end business processes? 
- How to close the gap between business requirements and business process design and the implementation of ERP applications?

- How to make sure that blueprint documentation represents the actual implementation?

- How to improve the communication of IT experts and end-users during and after the implementation?

Business process modelling builds a bridge between business and IT. It introduces a process culture and reduces conflicts during an ERP implementation. A rapidly growing number of frameworks and modelling tools have been developed for an integrated modelling of the entire enterprise with a focus on both organisational modelling and information systems modelling (Giaglis et al., 2005). A number of the well known methodologies for ERP systems implementation include some business process modelling, however, in a very limited scope. For example, the ASAP methodology (Accelerated SAP) contains aligns the software (at a high level) with the internal business processes phase, yet in the form of interviews and workshops (Gulledge, Simon, 2005). In practice this phase mostly leads to fulfilling wishes and lacks a strategic view. A successful ERP project is hardly ever possible without a business process change that requires its wider scope.

The results of this investigation showed that less than half of the companies that implement an ERP system perform any kind of business modelling to asses their information needs. This is rather worrying, and shows that the importance of business process modelling in ERP implementation projects is still not acknowledged in practice. Moreover, even the idea of conformation between an ERP system and business processes in the company has been poorly accepted in practice.

Models developed in the initial stages of the proposed framework are valuable for companies for other purposes as well. People start to be aware that their work is part of something that gives value to the customer. Any kind of business process modelling increases coordination among departments and other aspects of business process maturity (McCormack, 2007). The knowledge created during ERP implementation and management is a significant resource for a company. Business process modelling could be considered an important contribution and approach to the process of knowledge management, since business process repositories could be used for knowledge creation, sharing and distribution (Apshavalka and Grundspenkis, 2003).
On the other hand, there are also some threats in business process modelling. First of all, a too detailed process modelling can delay the project. Besides, the selection of the proper modelling technique and tools is also important. As is evident from our previous research (Jaklič et al, 2006), we suggest not to select every tool that enables modelling with BPMN or similar techniques in this case.

The goal of the research was to build a framework of ERP implementation and the authors have taken steps toward addressing this goal. This paper provided valuable insights towards understanding the role of business process modelling in implementing an ERP system. The framework was established and discussed. Finally, it has been applied to various ongoing projects in Slovenian companies. The results from business practice provide a foundation for further empirical studies. At present, its development is not completed, and in order to verify and validate the proposed framework the authors intend to continue this research. Consequently, the authors plan to explore these issues through further research. $[$.

\section{References}

A. Kovačič, and V. Bosilj-Vukšić, "Management poslovnih procesov", GV založba, Ljubljana, 2005. In Slovene.

Al-Mashari, M. 2003. A Process Change-Oriented Model for ERP Application, International Journal of Human-computer Interaction 16(1): 39-55.

Apshavalka, D. and Grundspenkis, J. 2003. Making organisations to act more intelligently in the framework of organisational knowledge management system. Scientific Proceedings of Riga Technical University on Computer Science RTU 17: 72-82.

Arif, M., Kulonda D., Jones, J. and Proctor, M. 2005. Enterprise information systems: technology first or process first?, Business Process Management Journal 11(1): 5-21.

Avison, D. and Fitzgerlad, G. 2003. Where now for development methodologies. Communications of the ACM 46 (1): 78-82.

Boykin, R.F. 2001. Enterprise resource-planning software: a solution to the return material authorization problem. Computers in Industry 45: 99-109.

Bozarth, C. 2006. ERP implementation efforts at three firms: Integrating lessons from the SISP and IT-enabled change literature. International Journal of Operations \& Production Management 26(11): 1223-1239.

Chen, I.J. 2001. Planning for ERP systems: analysis and future trend. Business Process Management Journal 7(5): 374-86.

Davenport, T. 1998. Putting the enterprise into the enterprise system. Harvard Business Review July-August 1998: 121-131.

Ehie, I.C. and Madsen, M. 2005. Identifying critical issues in enterprise resource planning (ERP) implementation. Computers in Industry 56(6): 545-557. 
Frye, D.W. and Gulledge, T.R. 2007. End-to-end business process scenarios. Industrial management \& Data Systems 107(6): 749-761.

Garcia-Sanchez, N. and Perez-Bernal, L. 2007. Determination of Critical Success Factors in Implementing an ERP System: A Field Study in Mexican Enterprises. Information Technology for Development 13(3): 293-309.

Gargeya, V.B. and Brady, C. 2005. Success and failure factors of adopting SAP in ERP system implementation. Business Process Management Journal 11(5): 501-516.

Genovese, Y. 2005. Gartner Evaluates SAP: Treading Beyond Business Applications. Paper presented at the Symposium/ITxpo 2005. Moscone Center West, San Francisco, California: 15-19.

Giaglis, G., Hlupic, V., Vreede, G., and Verbraeck, A. 2005. Synchronous design of business processes and information systems using dynamic process modelling. Business Process Management Journal 11(5): 488500.

Gulledge, T. and Simon, G. 2005. The evolution of SAP implementation environments: A case study from a complex public sector project. Industrial Management \& Data Systems 105(6): 714-736.

Haines, M.N. and Goodhue, D.L. 2003. Implementation Partner Involvement and Knowledge Transfer in the Context of ERP Implementations. International Journal of Human-Computer Interaction 16(1): 23-28.

Huang, A., Yen, D., Chou, D. and Xu, U. 2003. Corporate applications integration: challenges, opportunities and implementation strategies. Journal of Business and Management 9(2): 137-145.

Huang, S, Chang, I, Li, S. and Lin, M. 2004. Assessing risk in ERP projects: identify and prioritize the factors. Industrial Management \& Data Systems 104(8): 681-688.

Jaklič, J., Bosilj Vukšić, V. and Indihar Štemberger, M. 2006. Business process oriented tool selection model - a case study. Paper presented at the conference Future challenges and current issues in business information, organisation and process management. Westminster Business School: 94-102.

Kim, Y., Lee, Z. and Gosain, S. 2005. Impediments to successful ERP implementation process. Business Process Management Journal 11(2): 158-170.

Koh, S.C.L. and Saad, S.M. 2006. Managing uncertainty in ERPcontrolled manufacturing environments in SMEs. International Journal of Production Economics 101(1): 109-127.

Loh, T.C. and Koh, S.C.L. 2004. Critical elements for successful ERP implementation in SMESs. International Journal of Production Research 42: 3433-3455.

McCormack, K. 2007. Business Process Maturity: Theory and Application.

META Group. 2004. Market Research: The State of ERP Services (Executive Summary). META Group, Inc. Stamford, CT.

Motwani, J., Subramanian, R. and Gopalakrishna, P. 2005. Critical factors for successful ERP implementation: Exploratory findings from four case studies. Computers in Industry 56: 529-544.

Nah, F., Lau, J. and Kuang, J. 2001. Critical factors for successful implementation of enterprise systems. Business Process Management Journal 7: 285-296.

Nah, F.F., Zuckweiler, K.M. and Lau, J.L. 2003. ERP Implementation: Chief Information Officers' Perceptions of Critical Success Factors. International Journal of Human-Computer Interaction 16(1) 5-22.
Shehab, E.M., Sharp, M.W., Supramaniam, L. and Spedding, T.A. 2004. Enterprise resource planning - an integrative review. Business Process Management Journal 10(4): 359-386.

Soh, C., Kien, S.S. and Tay-Yap, J. 2000. Cultural fits and misfits: is ERP a universal solution? Communications of the ACM 43(4): 47-51.

Stolovitsky, N. 2006. The Evolution of Enterprise Resource Planning Includes Service Industries. Technology Evaluation, Centers. http://www.technologyevaluation.com (accessed December 2007).

van der Aalst, W.M.P. and Weijters 2004. Process mining: a research agenda. Computers in Industry 53(3): 231-244.

Volkoff, O. 1999. Using the structurational model of technology to analyze an ERP implementation. Proceedings of the Americas Conference on Information Systems: 235-237.

Wei, C., Chien, C. and Wang, M.J. 2005. An AHP-based approach to ERP system selection. International Journal of Production Economics 96: 4762.

Woo, H.S. 2007. Critical success factors for implementing ERP: the case of $y$ Chinese electronics manufacturer. Journal of Manufacturing Technology Management 18(4): 431-442.

Woo, H.S. 2007. Critical success factors for implementing ERP: the case of a Chinese electronics manufacturer. Journal of Manufacturing Technology Management 18(4): 431-442. 